\title{
Retropharyngeal Abscess Managed With Ketamine Case Report
}

\author{
Kirti Saxena N* \\ Department of Anaesthesiology and Intensive care, Maulana Azad medical college and assoc. Lok Nayak Hospital, India
}

*Corresponding author: Dr. Kirti N Saxena, MD, Director, Professor, Department of Anaesthesiology and Intensive care, Maulana Azad medical college and assoc. Lok Nayak Hospital, New Delhi, India

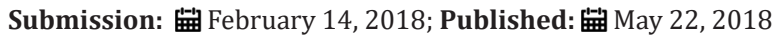

\begin{abstract}
A one year old male child presented to the pediatrics emergency with difficulty in breathing for the last 10-12 days. Severe inspiratory stridor was present and the accessory muscles of respiration were active. X-ray of lateral view of the neck showed increased soft tissue shadow in front of C2 vertebra suggestive of retropharyngeal abscess. Incision and drainage of retropharyngeal abscess was planned under general anesthesia after perforsming tracheostomy, as an emergency procedure. Intravenous glycopyrrolate 50 micrograms followed by $3 \mathrm{mg}$ of ketamine was given slowly till the patient became calm following which tracheotomy was carried out under local anesthesia achieved by infiltration with lignocaine by the otolaryngologist. After tracheotomy, the patient was given ketamine $5 \mathrm{mg}$ intravenously and oxygen: nitrous oxide (50:50) and Sevoflurane $2 \%$ through the tracheotomy tube attached to the breathing circuit with the patient breathing spontaneously. Incision and drainage of the abscess was carried out and $20 \mathrm{ml}$ of pus aspirated. We found ketamine to be very useful for sedation while carrying out a tracheotomy in this case.
\end{abstract}

Keywords: Retropharyngeal abscess; Ketamine; Obstructed airway

Implications

General anaesthesia in pediatric patients with airway obstruction is a challenge to the anesthesiologist. We describes the anesthetic management of a child with retropharygeal abscess who was having airway obstruction. Ketamine can be life saving in these situations

\section{Introduction}

Retropharyngeal abscess is a potentially fatal infection of the pharyngeal wall which is seen usually in the pediatric age group. It arises from suppurative infection of lymph nodes in the retropharyngeal space [1]. The commonest symptoms are fever, stridor, neck swelling and pharyngeal mass. Most of the children presenting with stridor are less than 1 year old [2]. Complications include airway obstruction and abscess rupture [1]. Tracheostomy may be required in some cases [3]. This is complicated by the fact that that tracheostomy in infants itself requires general anesthesia which is fraught with problems in these cases. We present a one year old child with retropharyngeal abscess who had to be anesthetized for tracheostomy followed by incision and drainage of the abscess.

\section{Case Report}

A one year old male child weighing $6.5 \mathrm{~kg}$ presented to the pediatrics emergency. The parents gave a history that there was difficulty in breathing for the last 10-12 days which increased on lying down and improved on sitting. There was also history of abnormal sound on crying however there was no history of foreign body ingestion. On examination the child was conscious but extremely restless. Severe inspiratory stridor was present and the accessory muscles of respiration were active .He was dehydrated, febrile and had poor general condition. His pulse rate was 160/ minute and respiratory rate was $30 /$ minute. On examination of the chest, there was decreased air entry on the left side with coarse crept in both lung bases. Chest X-ray showed opacities in the left lower zone but there was no foreign body. X-ray lateral view of the neck showed increased soft tissue shadow in front of C2 vertebra suggestive of retropharyngeal abscess.

Incision and drainage of retropharyngeal abscess after performing a tracheostomy was planned under general anesthesia as an emergency procedure and the child was taken into the operation theatre.Pulse oximetry showed a saturation of $70 \%$ but was not confirmatory as the child was moving. Cardiac monitor was attached and patient was given $100 \%$ oxygen through a face mask with capnograph attached to a pediatric breathing circuit. After a few minutes, the oxygen saturation increased to $96 \%$. Intravenous access was secured with a $24 \mathrm{G}$ cannula and $100 \mathrm{ml}$ of Ringer Lactate solution was given to correct dehydration. Intravenous glycopyrrolate 50 micrograms followed by $3 \mathrm{mg}$ of ketamine were 
given slowly till the patient became calm and sedated. Tracheostomy was carried out under local anesthesia achieved by infiltration with lignocaine by the otolaryngologist. After tracheostomy, the patient was given ketamine $5 \mathrm{mg}$ intravenously and oxygen:nitrous oxide (50:50) and sevoflurane 2\% through the tracheostomy tube attached to the breathing circuit with the patient breathing spontaneously. Incision and drainage of the abscess was carried out and $20 \mathrm{ml}$ of pus aspirated. The patient was reversed with $100 \%$ oxygen.

Postoperatively, the patient was sedated but responding to stimuli. His pulse rate was $110 / \mathrm{min}$ and he was maintaining an oxygen saturation of $99 \%$ with oxygen administered through a T-piece attached to the tracheostomy tube. There were no signs of airway obstruction. Thereafter the patient was kept in the pediatric ICU with the tracheostomy. He was administered antibiotics, and dexamethasone intravenously and kept on inhalation of nebulized adrenaline. He continued to do well and the tracheostomy tube was removed after seven days and the child could be discharged after two more days of observation.

\section{Discussion}

Retropharyngeal abscess is a potentially fatal infection of the posterior pharyngeal wall. The common presentation consists of fever, toxic appearance, stiffness of neck and respiratory distress. $\mathrm{X}$-Ray soft tissue neck in both anteroposterior and lateral view should be done to evaluate the extent of airway compromise. MRI and CT scan are preferred for diagnosis and evaluation of airway3 but it may not be possible to do these in airway emergencies. Also sedation is required for these procedures in children, which can suppress their respiratory drive leading to apnea.

There are many difficulties and challenges in the management of the pediatric airway for anaesthesiologists. General anesthesia is difficult in pediatric patients with airway anomalies with the threat of respiratory obstruction looming high. Tracheostomy or foreign body removal cannot be done under local anesthesia and needs general anesthesia. Upper airway surgery in children is usually performed with an endotracheal tube in place.

It is important to achieve airway control in awake state but because children do not cooperate for this so the awake but sedated approach is preferred [5]. The common sedatives used are short acting opiates and benzodiazepines for the relatively cooperative patients. For others general anesthesia may become necessary. Assisted spontaneous ventilation during anesthesia is the preferred technique and administration of muscle relaxants is avoided as it can lead to total obstruction. Even administration of volatile anaesthetics can result in respiratory arrest and hypoxaemia [1].

Retropharyngeal abscess causes upper airway obstruction and trismus and in our case was causing almost total obstruction of the airway. Intubation in these cases has been associated with difficulty as laryngoscopy and intubation both can rupture the abscess1 leading to further obstruction or aspiration. Fibreoptic bronchoscopy needs expertise and is time consuming even in experienced hands in case of airway emergencies especially in infants. It is difficult in cases with airway edema and congestion but has been used in an adult patient [5]. Tracheostomy is unavoidable if the abscess is large. General anesthesia is inevitable in such cases $[1,3]$. Death following obstruction has been reported under general anesthesia [3]. In our case it was difficult to give general anesthesia as the child was very small and in respiratory distress while the abscess was large. Inhalation of a mixture of Helium and Oxygen would be ideal but this was not available with us [6].

Ketamine produces both hypnosis and analgesia. It can be used alone or with midazolam for infants and young children. Ketamine in low doses of less than $1 \mathrm{mg} / \mathrm{kg}$ usually preserves spontaneous ventilation while providing sedation and analgesia [7]. It maintains the ventilatory response to carbon dioxide but large doses can lead to apnea. Upper airway skeletal muscle tone is well preserved. It should be administered slowly and titrated to prevent over sedation and apnea. Also since ketamine is a bronchodilator it helps in maintaining oxygenation in patients with chest infection such as ours [8]. It has been used in the management of difficult airway due to these properties $[9,10]$. An antisialogogue is recommended to decrease secretions

\section{References}

1. Rabb MF, Szmuk P (2007) The difficult pediatric airway in Benumof's Airway management. Published by Mosby $2^{\text {nd }}(e d n)$ 783-833

2. Coulthard M, Isaacs D (1991) Retropharyngeal abscess. Arch Dis Child 66: $1227-1230$.

3. Ameh EA (1999) Acute retropharyngeal abscess in children. Ann Trop Paediatr 19(1): 109-112.

4. Craig FW, Schunk JE (2003) Retropharyngeal abscess in children: clinical presentation, utility of imaging, and current management. Pediatrics 111(6 Pt 1): 1394-1398.

5. Pollard BA, El-Beheiry H (1999) Pott's disease with unstable cervical spine, retropharyngeal cold abscess and progressive airway obstruction. Can J Anesth 46(8): 772-775.

6. Grosz AH, Jacobs IN, Cho C, Schears GJ (2001) Use of helium oxygen mixtures to relieve upper airway obstruction in a pediatric population. The laryngoscope 111(9): 1512-1514.

7. Bahk JH, Sung J, Jang IJ (2002) A comparison of ketamine and lidocaine spray with propofol for the insertion of laryngeal mask airway in children: a double-blinded randomized trial. Anesth Analg 95(6): 15861589.

8. Kurdi MS, Theerth KA, Deva RS (2014) Ketamine: current applications in anesthesia, pain, and critical care. Anesth Essays Res 8(3): 283-290.

9. Paterson NA (2008) Management of an unusual pediatric difficult airway using ketamine as a sole agent. Paediatr Anaesth 18(8): 785-788.

10. Saraswat M, Radhakrishnan M (2001) Burns Contracture of Neck: Two Case Reports of Difficult Intubation. The Internet Journal of Anesthesiology 5(3). 
Creative Commons Attribution 4.0 International License

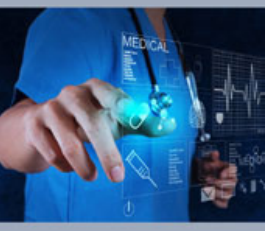

- High-level peer review and editorial services

- Freely accessible online immediately upon publication

- Authors retain the copyright to their work

- Licensing it under a Creative Commons license

- Visibility through different online platforms 